\title{
Management and decision-making strategy for systolic anterior motion after mitral valve repair
}

\author{
Giuseppe Crescenzi, MD, ${ }^{\mathrm{a}}$ Giovanni Landoni, MD, ${ }^{\mathrm{a}}$ Alberto Zangrillo, MD, ${ }^{\mathrm{a}}$ Fabio Guarracino, $\mathrm{MD},{ }^{\mathrm{c}}$ Concetta Rosica, MD, \\ Giovanni La Canna, MD, ${ }^{\mathrm{b}}$ and Ottavio Alfieri, $\mathrm{MD}^{\mathrm{b}}$
}

\begin{abstract}
Objective: Systolic anterior motion can complicate mitral valve repair. It can have no clinical consequence or cause low cardiac output syndrome and hypotension. The management of systolic anterior motion in the operating room remains controversial: some groups advocate nonsurgical management, and others propose immediate surgical correction. Conventional hemodynamic measures require time and can be unsuccessful. While describing our experience, we propose a simple and innovative management and classification of this complication.
\end{abstract}

\begin{abstract}
Methods: Presenting the data of 608 consecutive patients who underwent mitral valve repair for degenerative mitral valve disease, we describe a novel 2-step conservative management consisting of intravascular volume expansion and discontinuation of inotropic drug (step 1) and increasing afterload by means of ascending aortic manual compression while administering $\beta$-blockers (step 2). We also describe a novel classification of systolic anterior motion: easy to revert (responding to step 1), difficult to revert (responding to step 2), or persistent.
\end{abstract}

\begin{abstract}
Results: The overall incidence of systolic anterior motion was 9.8\% (60/608): 40 patients had easy-to-revert systolic anterior motion, and 15 had difficult-to-revert systolic anterior motion. Five patients had a persistent condition and underwent surgical intervention within 48 hours.
\end{abstract}

Conclusions: Systolic anterior motion after repair of a degenerative mitral valve is common. Surgical revision in the minority of patients unresponsive to standard conservative management is suggested.

Systolic anterior motion (SAM) is a possible complication after mitral valve (MV) repair. The degree of SAM extends along a continuous spectrum ranging from minor chordal protrusion without left ventricular outflow tract (LVOT) obstruction to more severe forms with LVOT obstruction, with variable clinical presentations from unaltered hemodynamics to low cardiac output syndrome and hypotension. The degree of SAM is considered mild when LVOT obstruction is absent and mitral regurgitation (MR) is absent or negligible; moderate when LVOT maximum pressure gradient ( $\triangle \mathrm{P}$ max) ranges between 20 and $50 \mathrm{mmHg}$, MR is judged to be mild to moderate, or both; and severe when LVOT $\Delta \mathrm{P}$ max is $50 \mathrm{~mm} \mathrm{Hg}$ or greater, MR is judged to be severe, or both. ${ }^{1}$

The management of SAM in the operating room remains controversial; this is mainly due to the absence of precise directions to guide decision-making strategy, with some groups advocating nonsurgical management ${ }^{1,2}$ and others proposing immediate surgical correction. ${ }^{3-5}$

From the Departments of Cardiothoracic Anesthesia and Intensive Care ${ }^{\mathrm{a}}$ and Cardiac Surgery, ${ }^{\mathrm{b}}$ Università Vita-Salute San Raffaele, Milano, Italia e Istituto Scientifico San Raffaele, Milan, Italy, and Cardiothoracic Anesthesia and ICU and Cardiac Surgery, ${ }^{\mathrm{c}}$ Azienda Ospedaliera Universitaria Pisana, Cisanello Hospital, Pisa, Italy.

Received for publication April 23, 2008; revisions received July 2, 2008; accepted for publication Aug 7, 2008.

Address for reprints: Giovanni Landoni, MD, Department of Cardiothoracic Anesthesia and Intensive Care, Istituto Scientifico San Raffaele, Milano, Italia, Via Olgettina 60 Milano, 20132 Italy (E-mail: landoni.giovanni@hsr.it)

J Thorac Cardiovasc Surg 2009;137:320-5

$0022-5223 / \$ 36.00$

Copyright (c) 2009 by The American Association for Thoracic Surgery

doi:10.1016/j.jtcvs.2008.08.018
We present the data of 608 consecutive patients undergoing MV repair for degenerative MR over a 2-year period. SAM was detected in 60 patients (study group) in whom a precise management protocol in the operating room was consistently and uniformly applied. The results are herein reported, and criteria for immediate reoperation (revision or valve replacement) are presented.

\section{MATERIALS AND METHODS}

\section{Clinical Material}

After ethics committee approval and patients' written consent, we collected data on 608 consecutive patients (mean age, $56 \pm 14$ years; $45 \%$ female subjects) who underwent MV repair for degenerative disease at San Raffaele University Hospital, Milan, Italy, over a 2-year period (February 2005 to March 2007). Patients with functional MV regurgitation, rheumatic heart disease, or hypertrophic obstructive cardiomyopathy were not included. A great variety of anatomic lesions and clinical conditions were present, with surgical indications being always adherent to the current guidelines. ${ }^{6,7}$ A wide spectrum of left ventricular end-diastolic diameter (mean \pm standard deviation, $56 \pm 7 \mathrm{~mm}$ ), left atrial size $(47 \pm 7 \mathrm{~mm})$, ejection fraction (59\% $\pm 7 \%$ ), and New York Heart Association (NYHA) class was included in this study. The majority of the patients $(57 \%)$ were classified as being in NYHA class I or II, whereas 39\% were in NYHA class III. Atrial fibrillation was present in a minority of patients $(21 \%)$. Patients submitted to tricuspid annuloplasty and concomitant coronary artery bypass grafting were enrolled. All patients had severe MR according to preoperative quantitative echocardiographic examination. ${ }^{6,7}$

\section{Surgical Repair}

The operation was carried out either through a midline sternotomy or with a minimally invasive approach using cardiopulmonary bypass (CPB), mild hypothermia, and cardioplegic arrest of the heart.

The valve was approached through the left atrium. Techniques of MV repair were applied according to the anatomic lesions responsible for MR. For posterior leaflet prolapse, a quadrangular resection was used. When 


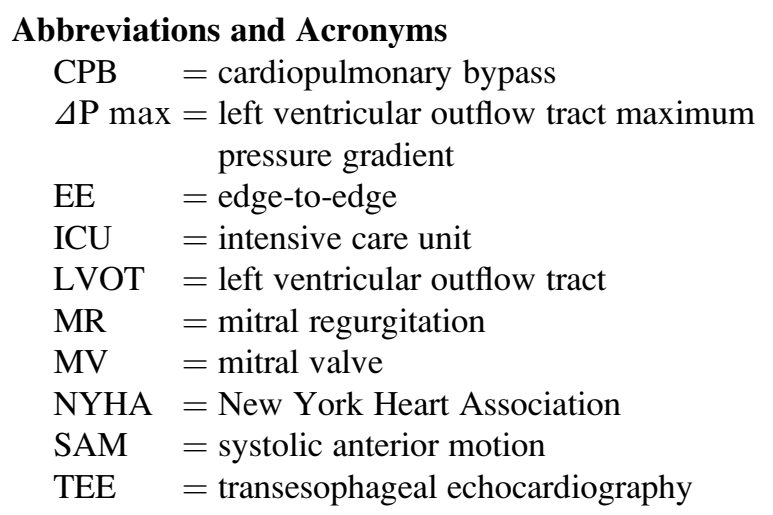

the height of the posterior leaflet was greater than $15 \mathrm{~mm}$, a sliding plasty was invariably associated. ${ }^{8}$ In anterior or bileaflet prolapse, the edgeto-edge (EE) technique, as described by our group, ${ }^{9,10}$ has been used. Occasionally, artificial chordae were used alone or in combination with the above-mentioned techniques. The type of mitral repair carried out is shown in Table 1.

A prosthetic ring was implanted in all cases. The appropriate ring size was chosen on the basis of the intertrigonal distance and the area of the anterior leaflet, according to the well-established techniques of mitral repair in degenerative mitral disease. ${ }^{11,12}$ In patient's with Barlow's disease, the appropriate ring size ranged between 36 and $40 \mathrm{~mm}$. A complete semirigid ring was used in 203 patients, and a partially flexible ring was used in 405 patients.

\section{Diagnosis of SAM}

In all patients intraoperative transesophageal echocardiographic (TEE) analysis was performed in the operating room immediately after interruption of CPB. SAM was defined as any portion of the MV leaflet or chordal structure protruding into the LVOT. ${ }^{13}$ Significant color Doppler aliasing in the LVOT was considered consistent with turbulent flow and obstruction. Velocities through the LVOT were measured with continuous-wave Doppler scanning. Concomitant MR was recorded. The degree of SAM was determined on the basis of the gradient across the LVOT and of the severity of MR, as described above.

\section{Management Protocol}

When some degree of SAM was diagnosed after CPB interruption, a well-defined management protocol was applied, including 2 consecutive steps (step 2 was applied if SAM was not solved by step 1). Step 1 was represented by established procedures, such as intravascular volume expansion and simultaneously discontinuation of any inotropic drug. Step 2 was represented by a maneuver intended to acutely increase the afterload (partial digital occlusion of the ascending aorta) and simultaneous administration of $\beta$-blockers (bolus of esmolol, $1 \mathrm{mg} / \mathrm{kg}$ ). ${ }^{14}$

The effect was invariably immediate and could be observed by means of echocardiographic analysis. Heart rate decreased significantly, and arterial blood pressure significantly increased.

Volume expansion was gradually performed over a period of a few minutes, allowing for adaptation and monitoring of pulmonary artery pressure. Discontinuation of inotropic drugs was performed in those few patients who inappropriately started them because of SAM-induced hypotension before performing postoperative TEE examination.

The compression after inspection and palpation of the ascending aorta acutely increases the afterload, and it was maintained for a 30 -second period. Simultaneously, esmolol was administered at a dose of $1 \mathrm{mg} / \mathrm{kg}$.

The goal of this conservative management in the operating room was to abolish SAM (absence of the LVOT obstruction and MR). SAM was classified according to its reversibility as easy to revert, difficult to revert, and persistent. SAM was defined as easy to revert when it disappeared only after intravascular volume expansion, discontinuation of any inotropic drug, or both; as difficult to revert when it disappeared after increasing afterload and administration of a bolus of esmolol $(1 \mathrm{mg} / \mathrm{kg})$; and as persistent if it did not disappear after conservative management.

If there was little or no improvement (SAM remaining at least moderate: $\Delta \mathrm{P}$ max in the LVOT ranges between 20 and $50 \mathrm{~mm} \mathrm{Hg}$, MR is graded as mild to moderate, or both) after conservative management, reoperation was immediately performed, or the patient was strictly monitored in the intensive care unit (ICU)

\section{Statistical Analysis}

Data were electronically stored, analyzed by use of Epi Info 2002 software (Centers for Disease Control and Prevention, Atlanta, Ga), and expressed as numbers and percentages. The $\chi^{2}$ test with the Yates correction or the Fisher's exact test was used when appropriate to compare the different surgical techniques in their relationship with the occurrence of SAM.

\section{RESULTS}

Immediately after CPB discontinuation, 60 (9.8\%) of 608 patients presented with SAM after MV repair (Table 1). SAM was more frequently reported after quadrangular resection without sliding than with other techniques. The incidence of SAM was statistically different according to

TABLE 1. Types of surgical repair techniques performed in 608 patients with myxomatous disease, leaflet prolapse, or flail

\begin{tabular}{|c|c|c|c|c|}
\hline $\begin{array}{l}\text { Type of surgical } \\
\text { repair technique }\end{array}$ & No. of patients & $\begin{array}{c}\text { No. }(\%) \text { with systolic } \\
\text { anterior motion }\end{array}$ & $\begin{array}{l}\text { Classification of systolic } \\
\text { anterior motion: } \\
\text { easy/difficult/persistent }\end{array}$ & Reoperation \\
\hline QR & 242 & $40(16.5 \%)$ & $29 / 8 / 3$ & 3 \\
\hline $\mathrm{QR}+$ sliding & 146 & $9(6.2 \%)$ & $4 / 3 / 2$ & 2 \\
\hline Central E-E & 146 & $9(6.2 \%)$ & $5 / 4 / 0$ & 0 \\
\hline Paracommissural E-E & 42 & 0 & 0 & 0 \\
\hline $\mathrm{AC}$ & 23 & $2(8.7 \%)$ & $2 / 0 / 0$ & 0 \\
\hline Other procedures & 9 & 0 & 0 & 0 \\
\hline Total & 608 & $60(9.8 \%)$ & $40 / 15 / 5$ & 5 \\
\hline
\end{tabular}

$Q R$, Quadrangular resection of the posterior mitral valve leaflet without the sliding technique; $Q R$ plus sliding, quadrangular resection of the posterior mitral valve leaflet with the sliding technique; central E-E, central edge-to-edge technique; paracommissural E-E, paracommissural edge-to-edge technique; $A C$, anterior leaflet procedures with artificial chordae. 


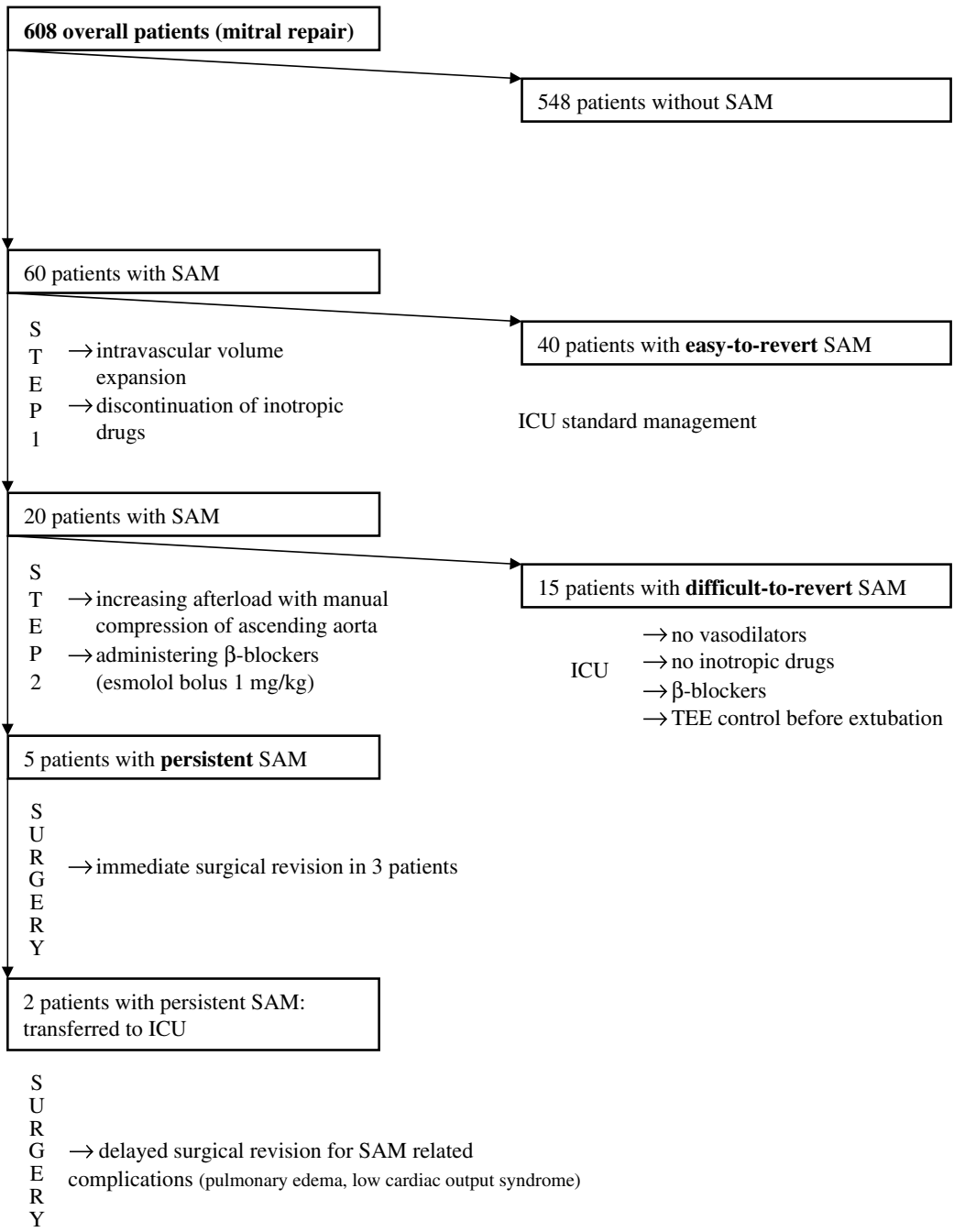

FIGURE 1. Management of 608 patients undergoing mitral repair for degenerative mitral valve disease (myxomatous disease, leaflet prolapse, or flail), focusing on postoperative systolic anterior motion (SAM). ICU, Intensive care unit; TEE, transesophageal echocardiography.

surgical techniques of MV repair $(P<.001)$, with the isolated quadrangular resection technique carrying a risk $(16.5 \%)$ significantly higher than that of the sliding technique $(6.1 \%, P=.005)$ and the EE technique $(6.1 \%, P=.005)$. No SAM was detected in the group with paracommissural EE repair. Furthermore, we noted that the distinctive feature of SAM after central EE repair $(9 / 146[6.1 \%])$ was the absence of severe MR also in case of severe LVOT obstruction.

Forty patients had easy-to-revert SAM (Figure 1), which disappeared with intravascular volume expansion and discontinuation of inotropic drugs. They were not treated with $\beta$-blockers. Fifteen patients had difficult-to-revert SAM that required afterload increase (by means of manual compression of the ascending aorta) and administration of $\beta$-blockers (bolus of esmolol, $1 \mathrm{mg} / \mathrm{kg}$ ). Five patients with persistent SAM not responding to conservative management required revision of repair or valve replacement.
Surgical reinterventions were performed either immediately (in 3 patients with a poor hemodynamic status) or within 48 hours (in 2 patients with a progressive deterioration of clinical conditions). Table 2 shows the details of the 5 patients submitted to re-repair or replacement.

The first 3 patients (A, B, and C) had a similar perioperative course with an immediate second pump run for revision of mitral repair, as described in detail in Table $2 .^{3}$

Patient D underwent a quadrangular resection of the posterior MV leaflet without sliding and had severe persistent SAM at TEE control, with modest improvement of obstruction ( $\triangle \mathrm{P}$ max ranging from $>50 \mathrm{~mm} \mathrm{Hg}$ to $50-20 \mathrm{~mm} \mathrm{Hg}$ ) and MR (graded moderate to severe) after conservative therapy. After an apparently uneventful immediate postoperative course, the patient had pulmonary edema 48 hours after the operation; TEE evaluation showed SAM with severe LVOT obstruction and MR that failed to improve with 
TABLE 2. Details of the $5(0.8 \%)$ of 608 patients with persistent systolic anterior motion (that did not subside or showed only a little improvement after conservative management)

\begin{tabular}{llcccccc}
\hline $\begin{array}{c}\text { Patient } \\
\text { ID }\end{array}$ & \multicolumn{1}{c}{$\begin{array}{c}\text { Surgical } \\
\text { technique }\end{array}$} & $\begin{array}{c}\text { Intraoperative } \\
\text { TEE control LVOT }\end{array}$ & $\begin{array}{c}\text { Intraoperative } \\
\text { TEE control of } \\
\text { mitral regurgitation }\end{array}$ & $\begin{array}{c}\text { After step 2 } \\
\text { conservative } \\
\text { treatment }\end{array}$ & $\begin{array}{c}\text { Surgical } \\
\text { revision }\end{array}$ & $\begin{array}{c}\text { New surgical } \\
\text { technique }\end{array}$ & $\begin{array}{c}\text { Complete } \\
\text { resolution }\end{array}$ \\
\hline A & QR & Severe obstruction & Severe & No improvement & Early & Sliding & Yes \\
B & QR & Severe obstruction & Severe & No improvement & Early & Sliding & yes \\
C & QR plus sliding & Severe obstruction & Severe & No improvement & Early & Edge to edge (1) (8) & yes \\
D & QR & Severe obstruction & Severe & Little improvement & Delayed & Sliding & Yes \\
E & QR plus Sliding & Severe obstruction & Severe & Little improvement & Delayed & Valve replacement & - \\
\hline
\end{tabular}

TEE, Transesophageal echocardiography; LVOT, left ventricular outflow tract; step 2 conservative treatment, afterload increase (ascending aorta manual compression) and $\beta$-blockers (bolus of esmolol, $1 \mathrm{mg} / \mathrm{kg}$ ); $Q R$, quadrangular resection of the posterior mitral valve leaflet without the sliding technique; $Q R$ plus sliding, quadrangular resection of the posterior mitral valve leaflet with the sliding technique.

medical therapy. A redo revision of the repair was decided (sliding resection), and SAM was resolved.

After a quadrangular resection of the posterior MV leaflet with sliding, patient $\mathrm{E}$ was very similar to patient $\mathrm{D}$ in the operating room, but he showed a severe low cardiac output syndrome while in the ICU. A TEE examination revealed severe SAM with severe LVOT obstruction and severe MR determining left ventricular apical ballooning, with balloon-like left ventricular motion abnormality and hypercontractile basal segments associated with ST-segment elevation on electrocardiographic analysis (the tako-tsubo syndrome). ${ }^{15,16} \mathrm{~A}$ valve replacement was performed.

Notably, both patients who had persistent SAM and did not undergo immediate surgical correction had postoperative SAM-related complications (pulmonary edema and low cardiac output syndrome) requiring valve re-repair or a replacement operation within 48 hours.

In none of the 60 patients considered as the study population was SAM detected at the echocardiographic control performed before discharge from the hospital.

\section{DISCUSSION}

SAM after MV repair in the setting of degenerative disease (myxomatous valve, leaflet prolapse, or flail) is a common postoperative complication: we reported an incidence of $9.8 \%$ in the overall studied population, which is in accordance with a previous study that reported an $11 \%$ incidence. ${ }^{1}$ In most cases (55/60 [92\%] in our experience) a 2-step conservative management (expanding intravascular volume and discontinuing any inotropic drug as a first step and, most importantly, increasing afterload through manual compression of the ascending aorta while administering an intravenous bolus of esmolol as a second step) resolved the SAM. Occasionally $(5 / 60[8 \%]$ patients with SAM and 5/608 [0.8\%] of the study's overall population), SAM persists after conservative management, and in such cases surgical revision should be promptly considered.

Although SAM is a well-known complication after MV repair, there are not clear guidelines for its treatment, and different attitudes in management have been advocated, ranging from prolonged medical treatment ${ }^{1,2}$ to immediate surgical reintervention. ${ }^{3-5}$

As a consecutive series in a high-volume cardiac surgery center with high experience in MV repair, our patients presented a large spectrum of anatomic lesions and clinical conditions. Our results with MV repair were recently published. ${ }^{17,18}$ The repair technique was varied according to the mitral pathology and institutional protocols. The size of the ring was chosen according to the standard criteria (intertrigonal distance and the area of the anterior leaflet). ${ }^{17,18}$

The original 2-step protocol aims at classifying SAM on the basis of severity and reversibility and has implications for further decision making regarding treatment. The acute increase in afterload, obtained through manual compression of the ascending aorta, is a very useful maneuver that has never been described before. Afterload increase caused by vasoconstrictors could require time, might be unsuccessful, and might cause drug-related side effects.

Similar to other reports, the incidence of SAM is a relatively common problem $(9.4 \%)$, but the intractable SAM requiring valve revision or replacement is very uncommon. This 2-step approach allowed a decision-making strategy that is quite appropriate, considering the outcome. With regard to patients who do not respond to step 2, surgeons should proceed to immediate revision. The 2 patients who required a late surgical revision underwent several clinical problems. Correct decision making in this setting has to take into account that a redo revision of repair or valve replacement after a few days implies an increased perioperative risk.

Our data support aggressive management with an immediate second pump run and valve repair or replacement in this minority of patients $(5 / 608[0.8 \%]$ in our experience) who have postoperative persistent SAM. Our hypothesis is that in these 5 patients who experienced failure of medical therapy, there was an important mismatch between the amount of valvular tissue and the MV orifice area after MV repair. Unfortunately, possibly because of the small number of patients, we have been unable to detect 
preoperative echocardiographic variables associated with a nonresponse to steps 1 and 2. Patients with equally severe SAM and similar preoperative echocardiographic findings had complete regression of SAM after the maneuvers.

Although previous studies have stressed the "benign", long-term evolution of SAM, recent articles report the need for surgical revision within the first postoperative days for patients who had SAM early after mitral repair in the operating room and were conservatively managed. ${ }^{4}$ Two cases of late failure of MV repair caused by SAM have been reported, ${ }^{19-21}$ and these required reoperation.

According to Filsoufi and Carpentier, ${ }^{22}$ we think that " one the most remarkable values of reconstructive valve surgery is to allow the patient to enjoy a normal life without medications and, in many instances, to be cured for the rest of his life.' Many patients in our case series were in NYHA class I or II preoperatively, and to discharge them postoperatively with chronic $\beta$-blockers because of SAM could be assimilated to surgical failure.

Our data support the management that has been consistently applied in our institution during the study period. Our study, focusing on those patients undergoing mitral repair who are at highest risk for postoperative SAM (myxomatous disease, leaflet prolapse, or flail) can only be compared with that of Brown and colleagues, ${ }^{1}$ who reported an incidence of SAM of $11 \%$ (with respect to our $9.8 \%$ ). A retrospective chart review of 1589 patients undergoing MV repair at the Mayo Clinic over a 10-year period identified 174 patients with early intraoperative SAM who were conservatively managed and required revision of repair or valve replacement in 4 cases $(2.3 \%$ of patients with SAM compared with our $8 \%$ ): 2 of their patients underwent an immediate operation, whereas the other 2 underwent operations within the first 4 postoperative weeks for SAM-related complications, one for pulmonary edema and the other for SAM coupled with an LVOT obstruction gradient of $100 \mathrm{~mm} \mathrm{Hg}$ and hemolytic anemia.

Notably, $62(36 \%)$ of 174 of their patients had SAM at hospital discharge. This discordant result could be partially due to the fact that we aim at obtaining complete reversibility of SAM before hospital discharge ${ }^{22}$ and in part to the different surgical techniques (quadrangular resection of the posterior MV leaflet with sliding technique and the central EE technique) we performed in many patients.

In this article we present, for the first time, a classification of SAM based on its reversibility to various maneuvers performed in sequence and indicate the percentage of patients responding to steps 1 and 2. Our classification also has relevant implications in regard to the medical management during ICU stay and hospitalization: whereas patients with easy-to-revert SAM require standard treatment, patients with difficult-to-revert SAM should not receive vasodilators but rather should receive $\beta$-blockers as soon as recovery from postoperative stunning is overcome, and in case of hypotension, they should receive a TEE examination and possibly be treated with intravenous fluids and not with inotropic drugs. A TEE examination before extubation is recommended. Patients with persistent SAM should be immediately surgically treated.

In this experience we have also described the occurrence of SAM after the EE technique, although it was never associated with significant MR.

\section{Limitations of the Study}

This study was not intended to investigate factors contributing to postoperative SAM after MV repair because the mechanisms of SAM and the risk factors involved are well recognized and described in the literature. Correlations between anatomic factors and the occurrence of SAM were not investigated in the present article. We described a simple management protocol consistently used in our institution to establish a clinically useful strategy. Data related to mortality and morbidity have not been reported. Our group has recently published results on MV repair, and this study is exclusively focusing on SAM. Finally, patients have been investigated only during the hospital stay, and no longterm data are reported. SAM is, however, more likely to develop immediately after surgical intervention (inotropic state of the heart), and it is unlikely that patients discharged without SAM had this complication later.

The low incidence of surgical revisions for SAM in this experience does not justify any change in our policy of adopting the quadrangular resection for posterior leaflet prolapse. We are considering being a bit more liberal in using the sliding plasty, which in this series was only limited to patients with a posterior leaflet height of greater than $15 \mathrm{~mm}$.

\section{CONCLUSIONS}

SAM after mitral repair for degenerative MV disease is a relatively frequent complication with heterogenous anatomic features and clinical manifestations; nonetheless, it responds to a number of maneuvers. A well-defined standardized management protocol consisting of a 2-step conservative maneuver has been suggested. Thereafter, patients requiring immediate revision can be easily identified. The validity of our strategy was demonstrated by the fact that in no patients was SAM detected at discharge.

\section{References}

1. Brown ML, Abel MD, Click RL, Morford RG, Dearani JA, Sundt TM, et al. Systolic anterior motion after mitral valve repair: is surgical intervention necessary? J Thorac Cardiovasc Surg. 2007;133:136-43.

2. Morford RG, Abel MD, Schaff HV, Click RL. Systolic anterior motion of the mitral valve after surgical repair: incidence and natural history. J Am Coll Cardiol. 2003;41:503.

3. Mascagni R, Al Attar N, Lamarra M, Calvi S, Tripodi A, Mebazaa A, et al. Edgeto-edge technique to treat post-mitral valve repair systolic anterior motion and left ventricular outflow tract obstruction. Ann Thorac Surg. 2005;79:471-3.

4. Charls LM. SAM-systolic anterior motion of the anterior mitral valve leaflet postsurgical mitral valve repair. Heart Lung. 2003;32:402-6. 
5. Brinster DR, Unic D, D'Ambra MN, Nathan N, Cohn LH. Midterm results of the edge-to-edge technique for complex mitral valve repair. Ann Thorac Surg. 2006; 81:1612-7.

6. Enriquez-Sarano M, Avierinos JF, Messika-Zeitoun D, Detaint D, Capps M, Nkomo V, et al. Quantitative determinants of the outcome of asymptomatic mitral regurgitation. $N$ Engl J Med. 2005;352:875-83.

7. Otto CM, Salerno CT. Timing of surgery in asymptomatic mitral regurgitation. $N$ Engl J Med. 2005;352:928-9.

8. Jebara VA, Mihaileanu S, Acar C, Brizard C, Grare P, Latremouille C, et al. Left ventricular outflow tract obstruction after mitral valve repair. Results of the sliding leaflet technique. Circulation. 1993;88(suppl):II30-4.

9. Alfieri O, Maisano F. An effective technique to correct anterior mitral leaflet prolapse. J Card Surg. 1999;14:468-70.

10. Alfieri O, Maisano F, De Bonis M, Stefano PL, Torracca L, Oppizzi M, et al. The double-orifice technique in mitral valve repair. A simple solution for complex problems. J Thorac Cardiovasc Surg. 2001;122:674-81.

11. Adams DH, Anyanwu AC, Rahmanian PB, Abascal V, Salzberg SP, Filsoufi F. Large annuloplasty rings facilitate mitral valve repair in Barlow's disease. Ann Thorac Surg. 2006;82:2096-101.

12. Mihaileanu S. Outflow tract obstruction and failed mitral valve repair. Circulation. 1994;90:1107-8.

13. Maslow AD, Regan MM, Haering JM, Johnson RG, Levine RA. Echocardiographic predictors of left ventricular outflow tract obstruction and systolic anterior motion of the mitral valve after mitral valve reconstruction for myxomatous valve disease. J Am Coll Cardiol. 1999;34:2096-104.
14. Crescenzi G, Rosica C, Marino G, Serini SM, Covello RD, Landoni G, et al. The use of esmolol to treat systolic anterior motion of the mitral valve after mitral valve repair. Eur J Anesthesiol. 2008;25:342-3.

15. Rosenmann D, Balkin J, Butnaru A, Wanderman K, Klutstein M, Tzivoni D Transient left ventricular apical ballooning. Report of 5 Israeli patients. Cardiology. 2006; 105:124-7.

16. Ionescu A. Subaortic dynamic obstruction: a contributing factor to hemodynamic instability in tako-tsubo syndrome? Eur J Echocardiogr. 2008;9:384-5.

17. De Bonis M, Lorusso R, Lapenna E, Kassem S, De Cicco G, Torracca L, et al Similar long-term results of mitral valve repair for anterior compared with posterior leaflet prolapse. J Thorac Cardiovasc Surg. 2006;131:364-70.

18. Maisano F, Caldarola A, Blasio A, De Bonis M, La Canna G, Alfieri O. Midterm results of edge-to-edge mitral valve repair without annuloplasty. J Thorac Cardiovasc Surg. 2003;126:1987-97.

19. Rescigno G, Cecconi M, Matteucci ML, Domenella P, Münch C, Iacobone G. Delayed systolic anterior motion after mitral valve repair. J Heart Valve Dis. 2006;15:512-4.

20. Doguet F, Zegdi R, Garçon P, Noghin M, Latrèmouille C, Chavaud S, et al. Systolic anterior motion (SAM): a rare cause of late failure in mitral valve repair. Arch Mal Coeur Vaiss. 2006;99:928-31.

21. Zegdi R, Carpentier A, Doguet F, Berrebi A, Khabbaz Z, Chauvaud S, et al. Systolic anterior motion after mitral valve repair: an exceptional cause of late failure. J Thorac Cardiovasc Surg. 2005;130:1453-4.

22. Filsoufi F, Carpentier A. Systolic anterior motion of the mitral valve. J Thorac Cardiovasc Surg. 2007;134:265-6. 